behaves differently from that in other parts of Britain. Seasonal trends have important implications in the planning of therapeutic trials and may help to identify environmental trigger factors in childhood asthma. A further study of national data is warranted.

We thank the Hospital Activity Analysis and statistical staff of the South East Thames region for their help.

1 Booth S, DeGroot IDO, Markush R, Horton RSM. Detection of asthma epidemics in seven cities. Arch Environ Health 1965;10:152.

2 Derrick EH. Asthma and the Brisbane climate. Aust NZ $\mathcal{F}$ Med 1972;3 : 235.

${ }^{3}$ Tromp SW. Influence of weather and climate on asthma and bronchitis. Review of Allergy 1968;22:1027.

${ }^{4}$ Anderson HR. Increase in hospitalisation for childhood asthma. Arch Dis Child 1978;53:295.

(Accepted 17 June 1983)

Royal Alexandra Hospital for Sick Children, Brighton BN1 3JN ALEX KHOT, $\mathrm{MB}, \mathrm{DCH}$, research associate

NIGEL EVANS, MRCP, DCH, consultant paediatrician

WARREN LENNEY, MD, MRCP, consultant paediatrician

Correspondence to: $\mathrm{Dr} W$ Lenney.

\section{Non-steroidal anti-inflammatory drugs and oral lichenoid reactions}

The aetiology of lichen planus is unknown. Stress, autoimmunity, underlying mesodermal disease, and infective agents have all been suggested as possible causes but have not gained wide support. Many drugs can provoke eruptions that clinically and histologically are similar to or even identical with lichen planus. Such lichenoid reactions may be caused by, for example, gold salts, methyldopa, quinine, and beta-blockers ${ }^{1}$ and may affect mucosal or cutaneous sites, either alone or together.

We describe a probable association between treatment with non-steroidal anti-inflammatory drugs and oral lichenoid reaction.

\section{Case report}

A 64 year old man presented to the oral medicine clinic, Birmingham Dental Hospital, with a five week history of soreness, white areas, and ulceration of the buccal mucosa. There was no history of skin, ocular, or genital lesions. He had been taking indomethacin for rheumatoid arthritis for 10 months. No other drug had been prescribed for 18 months.

Intraorally there were bilateral shallow erosions of the buccal mucosa surrounded by a network of lacy white striae typical of erosive lichen planus. There was no clinical evidence of arthropathy associated oral ulceration, including Behçet's syndrome, ulcerative colitis, or Reiter's syndrome.

Biopsy of the buccal mucosa showed atrophic parakeratinised oral epithelium with liquefaction degeneration of the basal cell layer. There was a band-like infiltrate of lymphocytes and histiocytes in the lamina propria immediately beneath the epithelium. These features are typical of oral lichen planus. Blood count and biochemical profile were normal.

Local treatment, initially with chlorhexidine and later with prednisolone mouthwash, provided no relief; indeed, the erosions became more widespread. Ibuprofen was substituted for indomethacin with minimal oral improvement. All medication was withdrawn and a short course of systemic steroids instituted. Within two weeks the oral ulceration had resolved. Because of increasing arthritic pain indomethacin suppositories were prescribed. Excellent relief of arthritic pain was produced but oral erosions recurred. The erosions did not respond to prednisolone mouthwashes but when the rectal indomethacin was discontinued they healed within 10 days. Fenclofenac and diflunisal were later prescribed, but these also produced oral erosion, which healed rapidly on withdrawal of the drugs. Soluble aspirin and aspirin-paracetamol combinations were prescribed, and the oral erosions resolved, with fading of the lichenoid striae. This improvement was maintained for about six months, but because of increasing arthritic pain the patient was prescribed flurbiprofen. Oral erosions returned and the lichenoid striae became very pronounced once again. These persisted essentially unchanged while the patient continued to take flurbiprofen.

\section{Comment}

These observations strongly suggest that indomethacin provoked the initial oral lichenoid eruption. The rapid recurrence of oral erosions on rechallenge with both indomethacin and other nonsteroidal anti-inflammatory agents and their equally rapid resolution on drug withdrawal indicate a causal relation between non-steroidal anti-inflammatory agents in general and oral lichenoid reactions. These drugs are known to be associated with non-specific oral ulceration but there is no previous documentation implicating them in oral lichenoid reactions.

The Committee on the Safety of Medicines has received a few reports of lichenoid reactions associated with non-steroidal antiinflammatory drugs (West Midlands Adverse Drug Reaction Monitoring Centre, personal communication).

On reviewing the records of 75 patients with oral lichen planus attending Birmingham Dental School's oral medicine clinic it was apparent that 20 of these were receiving non-steroidal anti-inflammatory drugs at the time of presentation. Clearly such an association in many of these patients will be coincidental, reflecting similar population groups susceptible to lichen planus and arthritic disorders. Within the group, however, there were seven patients whose oral lichenoid erosions resolved when the drugs were withdrawn. Two of them were rechallenged with the offending drug, producing a recurrence. In four other patients ibuprofen, fenbufen, sulindac, and benoxaprofen may have provoked oral lichenoid reactions.

In our patient there was a delay between beginning medication and the onset of oral eruptions. Holt and Navaratnam described such a phenomenon in methyldopa induced lichenoid eruptions. ${ }^{2}$ On withdrawal and rechallenge with this drug, however, the lichenoid eruptions resolved and recurred more rapidly. The eruptions provoked by non-steroidal anti-inflammatory drugs appear to behave similarly, recurrence of lichenoid reaction occurring within six days after rechallenge with fenbufen in one patient.

A controlled study is now under way to elucidate further this possible drug reaction.

Thanks are due to Professor H D Edmondson, Professor R M Browne, and $\mathrm{Mr} \mathrm{J}$ W Frame, whose patients formed the basis of this report. We also acknowledge the help of Dr L Beeley and the West Midlands Adverse Drug Reaction Monitoring Centre.

${ }^{1}$ Duxbury AJ. Systemic pharmacotherapy. In: Jones JH, Mason DK, eds. Oral manifestations of systemic disease. London: WB Saunders Co Ltd, 1980:454-89.

${ }^{2}$ Holt PJA, Navaratnam A. Lichenoid eruption due to methyldopa. $\mathrm{Br}$ Med F 1974;iii :234.

(Accepted 17 fune 1983)

The Dental School, St Chad's Queensway, Birmingham B4 6NN J HAMBURGER, BDS, MSC, department of oral surgery and oral medicine A J C POTTS, BDS, MRCPATH, department of oral pathology

Correspondence to: $\mathrm{Mr} \mathrm{J}$ Hamburger.

\section{Double blind cross over trial of ipratropium and placebo in chronic rhinorrhoea}

Watery rhinorrhoea in patients with perennial rhinitis responds poorly to treatment with topical beclamethasone diproprionate and disodium cromoglycate. ${ }^{1}$ Systemic antihistamines are effective in some cases but are associated with an unacceptable level of side effects. Ipratropium bromide, a topically active anticholinergic drug, inhibits rhinorrhoea induced by metacholine ${ }^{2}$ and produces symptomatic improvement in selected patients with perennial rhinorrhoea and abnormal reactivity to metacholine. ${ }^{3} \mathrm{We}$ assessed its use in unselected patients presenting with chronic watery rhinorrhoea as their main symptom.

\section{Patients, methods, and results}

Twenty men and women aged 18-78, mean 40 were referred consecutively to our clinic with chronic watery rhinorrhoea. All consented to the trial, completed a questionnaire on the nature and duration of symptoms, and underwent full otolaryngological examination, sinus radiology, and skin prick testing to common allergens. Rhinorrhoea had been present for one to 20 (mean five) years and lasted for a mean of $11 \cdot 1$ hours a day. Eight 
patients had a personal and four a family history of atopy. Four were smokers; except in these four tobacco smoke was the commonest physical agent provoking rhinorrhoea. Nineteen patients had previously received treatment with oral antihistamines, 18 with topical steroids, and nine with disodium cromoglycate, with no relief of symptoms. Seven patients gave one or more positive results on skin prick testing.

Placebo and active drug were delivered by indistinguishable coded pressurised aerosols fitted with nasal adaptors. The dosage of ipratropium was $40 \mu \mathrm{g}$ to each side of the nose four times a day. All other medication was withheld throughout the trial and the preceding two weeks. Patients were randomly allocated double blind to either ipratropium or placebo for two weeks and then crossed over to the other treatment for two weeks. Eleven patients received ipratropium first and nine placebo first.

Rhinorrhoea, nasal obstruction, and sneezing were assessed subjectively at the same time each day and rated on a four point scale $(0=$ absent, 3 =severe) during treatment and no treatment periods. Patients were interviewed at the start, midpoint, and end of the trial about overall treatment preference and side effects. Preference was analysed by the sign test and symptom scores by Wilcoxon's matched pairs signed rank test.

Fifteen patients preferred ipratropium and three preferred placebo $(p<0.01)$. Two had no preference. Scores for rhinorrhoea were significantly reduced during treatment with ipratropium compared with placebo ( $p<$ $0.01)$ and with no treatment $(p<0.01)$ (table). No significant difference was shown between the effect of placebo and ipratropium on nasal obstruction and sneezing, or between placebo and no treatment. No significant order effect was detectable. Three patients complained of a mild burning irritation in the nose when using ipratropium and four when using placebo.

Overall mean daily scores for symptoms assessed by 20 patients on four point scale $(0-a b s e n t, 3=$ severe $)$

\begin{tabular}{lccc} 
& Nasal obstruction & Rhinorrhoea & Sneezing \\
\hline No treatment & 1.04 & 2.06 & 1.05 \\
Placebo & 0.96 & 1.98 & 1.06 \\
Ipratropium & 1.22 & 1.42 & 1.13 \\
\hline
\end{tabular}

\section{Comment}

The nasal glands are innervated by parasympathetic nerves, and excessive watery rhinorrhoea is due to relative parasympathetic hyperactivity. ${ }^{4}$ Ipratropium bromide is an anticholinergic with high topical activity. It has no apparent systemic activity when inhaled in a dose of $80 \mu \mathrm{g}$ four times daily. Mygind reviewed the physiological and pharmacological rationale of treatment with ipratropium and discussed its potential value. ${ }^{5}$

The patients in this study were chosen on the basis only of symptoms; age, duration of symptoms, and atopic history varied. Intranasal ipratropium produced significant symptomatic relief of chronic rhinorrhoea without troublesome side effects despite the previous failure of other currently available medical treatment. The scores for nasal obstruction were higher during treatment with ipratropium than with placebo, although the difference was not significant at the $5 \%$ level ( $\mathrm{p}=0.074)$.

This study suggests, therefore, that intranasal ipratropium may give symptomatic relief in a broad spectrum of patients with chronic watery rhinorrhoea.

We thank Mr K Muntarbhorn and Dr A Eyre-Brook of Boehringer Ingelheim for their help.

1 Wentges RTR. Allergic and vasomotor rhinitis. In: Maran ADG, Stell PM, eds. Clinical otolaryngology. Oxford: Blackwell, 1979:226-38.

${ }^{2}$ Borum P. Intranasal ipratropium. Inhibition of metacholine induced hypersecretion. Rhinology 1978;16:225-33.

${ }^{3}$ Borum P, Mygind N, Larsen FS. Intranasal ipratropium; a new treatment for perennial rhinitis. Clin Otolaryngol 1979;4:407-11.

+ Golding-Wood PH. Surgery of the pterygopalatine fossa. In: Ballantyne

$\mathrm{J}$, Groves J, eds. Scott-Brown's diseases of the ear, nose, and throat.

Vol 4. London: Butterworths, 1979:447-81.

5 Mygind N. Nasal allergy. Oxford: Blackwell, 1978:333-42.

(Accepted 21 fune 1983)

Royal National Throat, Nose, and Ear Hospital, London WC1X 8DA

N P vON HAACKE, FRCS, FRCSI, senior registrar in otolaryngology

V MOORE-GILLON, FRCS, FRCSED, registrar in otolaryngology

L H CAPEL, MD, FRCP, consultant physician, allergy clinic

Correspondence to: $\mathrm{Mr}$ N P von Haacke, Department of Otolaryngology, City Hospital, Edinburgh EH10 5SB).

\section{Skull radiology in patients with psychiatric illness}

$X$ ray examinations are expensive and potentially dangerous. Evidence shows that diagnostic $x$ ray examinations are overused..$^{1-4} \mathrm{We}$ investigated the value of skull $x$ ray examination, isotope scanning, and computed tomography in patients with psychiatric illness.

\section{Patients, methods, and results}

The names of all patients admitted to the department of psychiatry, Royal South Hants Hospital, who had undergone skull $x$ ray examination, radioisotope scanning, and computed tomography between 23 May 1979, when the department opened, and 30 April 1981 were obtained from the daily records of the radiology departments of the Royal South Hants and Southampton General hospitals. Patients aged over 65 were excluded since another hospital treats nearly all patients with psychiatric illness in that age group. The clinical details of patients and the radiologists' reports were obtained from patients' case notes.

The 1691 admissions during this period generated 111 skull $x$ ray examinations, 16 isotope scans, and 13 computed tomograms. Two patients had undergone both isotope scanning and computed tomography.

Skull $x$ ray films showed abnormality in two patients. One had a schizophrenic illness with episodes of confusion and the other had had a stroke. In neither case did the radiological findings alter clinical management. Computed tomograms in both cases showed abnormality but an isotope scan in one did not. None of the 16 isotope scans showed any abnormality.

Four of the 13 computed tomograms showed abnormality. The scan findings supported the clinical diagnoses of presenile dementia in two patients and of a cerebral haemorrhage in the third. The fourth patient, who subsequently committed suicide, had an unusual schizophrenia with some organic features, and the scan findings were reported as "appearances probably represent a minor degree of (cerebral) atrophy and some atrophy of the superior vermis."

\section{Comment}

None of the skull $x$ ray films or computed tomograms disclosed an unsuspected abnormality. Furthermore, the four computed tomograms that showed abnormality confirmed the clinical diagnosis in three cases, and in the fourth the result was too vague to be of value. The findings did not alter clinical management in any appreciable way. The high proportion of cases in which skull $x$ ray examinations were performed $(7 \%$ ) suggests that some may be "routine." These findings are similar to those reported for chest $x$ ray examinations in patients with psychiatric illness from the same hospital. ${ }^{4}$

Our findings show that existing radiological techniques are not especially helpful in establishing a diagnosis in psychiatric disorder. There would have been little loss if the radiologists had refused to perform $x$ ray examinations on patients with psychiatric illness since any abnormality found was suspected on clinical grounds. Negative results may have had some value in excluding a suspected organic lesion or in reassuring the patient and relatives on this point. Our study, being retrospective, could not test that hypothesis.

The Royal College of Radiologists has conducted multicentre prospective inquiries into the value of $x$ ray procedures, producing useful results. The Royal College of Psychiatrists might consider a similar audit of $x$ ray procedures on patients with psychiatric illness.

' Rees AM, Roberts CJ, Bligh AS, Evans KT. Routine preoperative chest radiography in non-cardiopulmonary surgery. $\mathrm{Br}$ Med $\mathcal{F} 1976 ; \mathrm{i}: 1333-5$.

${ }^{2}$ Royal College of Radiologists. Preoperative chest radiology. Lancet 1979 ;ii:83-6.

${ }^{3}$ Royal College of Radiologists. A study of the utilisation of skull radiography in 9 accident-and-emergency units in the UK. Lancet 1980; ii:1234-7.

+ Hughes J, Barraclough BM. Value of routine chest radiography in psychiatric patients. Br Med $\mathcal{F} 1980 ; 281: 1461-2$.

(Accepted 1 fuly 1983)

Royal South Hants Hospital, Southampton SO9 4PE

SUDHIR C RASTOGI, MRCPSYCH, senior registrar

Department of Psychiatry, Faculty of Medicine, University of Southampton, Southampton SO9 4PE

BRIAN M BARRACLOUGH, FRACP, senior lecturer

Correspondence to: Dr B M Barraclough. 